\title{
Serum leptin in non-alcoholic fatty liver disease: Ambiguous clinical implications concerning cardiovascular disease
}

\author{
Dimitrios Patoulias ${ }^{1}$, Konstantinos Imprialos ${ }^{1}$, Konstantinos Stavropoulos ${ }^{1}$, and Michael Doumas ${ }^{1,2}$ \\ ${ }^{1}$ Second Propedeutic Department of Internal Medicine, Hippokration General Hospital, Aristotle University of Thessaloniki, Thessa- \\ Loniki, Greece; ${ }^{2}$ Veterans Affairs Medical Center, The George Washington University, Washington, D.C., WA, USA
}

\section{Dear Editor,}

We have read with great interest the results of the populationbased study performed by Rotundo et al., published in the previous issue of Clinical and Molecular Hepatology journal.' Researchers demonstrated that serum leptin levels were associated with the degree of hepatic steatosis -although the results became nonsignificant after adjustment for established cardio-metabolic risk factors, such as hypertension, type 2 diabetes, dyslipidemia, while they were also significantly associated with the degree of hepatic fibrosis, among patients with non-alcoholic fatty liver disease (NAFLD).

A previous meta-analysis of observational studies on 34,043 participants confirmed that NAFLD represents a "key player" in the field of cardiovascular disease (CVD), without clear and specific causality. ${ }^{2}$ A more recent observational study demonstrated that individuals with NAFLD exhibit a significantly higher risk of 10-year CVD incidence, further confirming this association. ${ }^{3}$ Thus, there is a direct need for the identification and establishment of novel markers that can prove useful for the diagnosis and prognosis of CVD in this sensitive population.

Top-level evidence has failed to demonstrate a significant association between circulating levels of leptin and CVD. More specifi- cally, Yang et al. showed that serum leptin was not significantly associated with coronary heart disease (odds ratio $[O R]=1.07$, $95 \%$ confidence interval $[\mathrm{Cl}] ; 0.96-1.19)$ and stroke $(\mathrm{OR}=0.98$, $95 \% \mathrm{Cl} ; 0.76-1.25) .{ }^{4}$ Similar results concerning coronary heart disease were also obtained from the meta-analysis performed by Chai et al. ${ }^{5}$ Researchers of the Multi-Ethnic Study of Atherosclerosis observed that serum leptin levels did not feature significant prognostic value, as far as the risk for incident CVD is concerned, as well. ${ }^{6}$ Thus, the role of leptin in CVD prediction is currently doubted.

However, it has been also demonstrated that liver fibrosis, but not liver steatosis, is associated with higher mortality among patients with NAFLD (hazard ratio $=1.69,95 \% \mathrm{Cl} ; 1.09-2.63$ ), determined by the NAFLD fibrosis score. Increase in mortality was almost entirely driven by cardiovascular causes, constituting liver fibrosis marker panels as predictors of CVD mortality among patients with NAFLD.' In the field of secondary prevention of CVD, newer data seem to agree with the aforementioned observations. $^{8}$

According to the data provided by Rotundo et al.', serum leptin might represent a novel measure of liver fibrosis. Thus, it may also be associated with CVD prediction in patients with NAFLD. Its po-

\section{Abbreviations:}

$\mathrm{Cl}$, confidence interval; CVD, cardiovascular disease; NAFLD, non-alcoholic fatty liver disease; OR, odds ratio

\section{Corresponding author: Dimitrios Patoulias}

Second Propedeutic Department of Internal Medicine, General Hospital

"Hippokration", Konstantinoupoleos 49, Thessaloniki 54642, Greece

Tel: +30-6946900777, Fax: +30-2310-225083

E-mail:dipatoulias@gmail.com

https://orcid.org/0000-0002-6899-684X 
tential use as a marker of liver fibrosis with direct clinical, prognostic implications, will elucidate the hypothesis, whether leptin has in fact a role in the prediction of CVD among patients with NAFLD, which constitutes a significant global health burden. ${ }^{9}$

\section{Authors' contribution}

Dimitrios Patoulias, Konstantinos Imprialos, and Konstantinos Stavropoulos wrote the manuscript. Michael Doumas critically reviewed the manuscript. All authors participated in final approval of the manuscript.

\section{Conflicts of Interest}

The authors have no conflicts to disclose.

\section{REFERENCES}

1. Rotundo L, Persaud A, Feurdean M, Ahlawat S, Kim HS. The Association of leptin with severity of non-alcoholic fatty liver disease: a population-based study. Clin Mol Hepatol 2018;24:392-401.

2. Targher G, Byrne CD, Lonardo A, Zoppini G, Barbui C. Non-alcoholic fatty liver disease and risk of incident cardiovascular disease: a meta-analysis. J Hepatol 2016;65:589-600.

3. Motamed N, Rabiee B, Poustchi H, Dehestani B, Hemasi GR, Khonsari MR, et al. Non-alcoholic fatty liver disease (NAFLD) and 10 year risk of cardiovascular diseases. Clin Res Hepatol Gastroenterol 2017:41:31-38.

4. Yang H, Guo W, Li J, Cao S, Zhang J, Pan J, et al. Leptin concentration and risk of coronary heart disease and stroke: a systematic review and meta-analysis. PLoS One 2017;12:e0166360.

5. Chai SB, Sun F, Nie XL, Wang J. Leptin and coronary heart disease: a systematic review and meta-analysis. Atherosclerosis 2014;233:310.

6. Martin SS, Blaha MJ, Muse ED, Qasim AN, Reilly MP, Blumenthal RS, et al. Leptin and incident cardiovascular disease: the Multi-ethnic Study of Atherosclerosis (MESA). Atherosclerosis 2015;239:67-72.

7. Kim D, Kim WR, Kim HJ, Therneau TM. Association between noninvasive fibrosis markers and mortality among adults with nonalcoholic fatty liver disease in the United States. Hepatology 2013;57:13571365.

8. Baik M, Kim SU, Kang S, Park HJ, Nam HS, Heo JH, et al. Liver fibrosis, not steatosis, associates with long-term outcomes in ischaemic stroke patients. Cerebrovasc Dis 2019;47:32-39.

9. Younossi Z, Anstee QM, Marietti M, Hardy T, Henry L, Eslam M, et al. Global burden of NAFLD and NASH: trends, predictions, risk factors and prevention. Nat Rev Gastroenterol Hepatol 2018;15:11-20. 